\title{
Jean-Claude Haelewyck : le parcours d'un chercheur devenu enseignant
}

Par

\section{Claude Obsomer}

Université de Namur, Université catholique de Louvain

$\mathrm{C}$

inq kilomètres à peine séparent Wasmes, son lieu de naissance, et Blaugies, son lieu de résidence. Une preuve que Jean-Claude Haelewyck reste fidèle à sa terre, le Borinage, qui le vit naître le mercredi 24 février 1952. Et pourtant c'est jusqu'à Jérusalem que ses pas le menèrent, tel le pèlerin du temps jadis, moins toutefois dans la quête spirituelle d'une (improbable) rédemption, que dans celle, toute intellectuelle, d'une meilleure compréhension des textes bibliques auquel il consacra son œuvre, voire sa vie.

Après des études secondaires au Collège Sainte-Marie de Saint-Ghislain, qui lui permirent de se former aux langues latine et grecque, il effectua deux années d'études au Séminaire de Namur, puis quatre autres au Séminaire de Tournai, avant de s'orienter vers une formation de type universitaire. C'est en 1976 qu'il s'inscrivit à l'Université catholique de Louvain, où il allait obtenir trois licences en quatre ans : théologie, philologie biblique, philologie et histoire orientales. Son mémoire, rédigé sous la direction du Père Bogaert, portait sur le chapitre 15 de la Genèse.

Ayant obtenu un mandat d'aspirant au FNRS dès 1980, il mena une recherche doctorale sur le texte lucianique du livre d'Esther, de nouveau sous la direction du Père Bogaert. C'est durant ce mandat qu'il fut appelé sous les drapeaux, effectuant son service militaire à l'Aumônerie du QG de Neder-over-Heembeek, où il collabora avec le futur évêque de Gand, Mgr Luysterman. Proclamé docteur en théologie en 1984, avec la plus grande distinction, il mena ensuite une carrière de chercheur au FNRS, devenant chargé de recherche en 1984, 
chercheur qualifié en 1988, maître de recherche en 2007, directeur de recherche en 2014 jusqu'à sa retraite en mars 2017.

Les publications nombreuses qui jalonnent ce cursus exemplaire furent consacrées notamment aux apocryphes de l'Ancien Testament, à la critique textuelle du Nouveau Testament, aux vieilles versions latines de la Bible, aux versions syriaques de Grégoire de Nazianze. Deux de ces thématiques sont liées à des projets collectifs auxquels il collabora au sein de l'Université catholique de Louvain : l'édition d'Isaïe dans le cadre du «Centre de recherches sur la Bible latine », de 1986 à 1992 ; l'étude des discours de Grégoire de Nazianze dans le cadre du « Groupe de recherches sur Grégoire de Nazianze », à partir de 1996.

C'est au cours de l'année académique 1993-94 qu'il effectua son séjour d'étude à l'École Biblique et Archéologique Française de Jérusalem. Grâce à un mémoire intitulé « David à Hébron », rédigé sous la direction de François Langlamet, il obtint son diplôme avec la mention très bien et les félicitations du jury.

À côté de cette activité de recherche foisonnante, Jean-Claude Haelewyck mena dès 1986 une carrière d'enseignant à l'Université catholique de Louvain. Attaché tantôt à la Faculté de Théologie, tantôt à la Faculté de Philosophie et Lettres (Institut orientaliste), il a cherché sans relâche à maintenir un pont entre les études bibliques et les études orientales, insistant auprès des étudiants de ces deux orientations sur l'importance d'étudier un texte dans sa langue d'origine. Il fut chargé d'enseigner l'« Introduction à l'Ancien Testament», la «Critique textuelle des textes bibliques », la « Grammaire comparée des langues sémitiques » (avec un manuel paru aux éditions Safran), mais aussi différentes langues sémitiques: l'araméen, l'hébreu, le phénicien, l'ougaritique. Nul doute que l'institution eût trouvé en lui un excellent professeur de syriaque, langue qu'il enseigna pendant plus de vingt ans dans le cadre d'une académie d'été française, l'« Académie des Langues Anciennes »(ALA), active en juillet à Saintes, Lyon, Lille, puis Digne.

Dans le domaine de la langue hébraïque, il dirigea deux thèses de doctorat : celle d'Axel Van de Sande en 2006, intitulée « Nouvelle perspective sur le système verbal de l'hébreu ancien : les formes qatala, yaqtul et yaqtulu », et celle de Naïma Afif en 2015, intitulée « Une version biblique du Coran en hébreu : la traduction d'Hermann Reckendorf (1857)».

C'est son expérience à l'ALA qui amena Jean-Claude Haelewyck à fonder l'« Académie Belge pour l'Étude des Langues Anciennes et Orientales " (ABELAO) ${ }^{1}$, pour laquelle il suscita la collaboration de l'auteur de ces lignes. Cette association sans but lucratif « a pour but de promouvoir l'enseignement et la recherche dans le domaine des langues anciennes et orientales, notamment par l'organisation de sessions de cours d'été, sur le site de l'Université de Louvain, à Louvain-la-Neuve ». La première session de cours eut lieu en août 2009 et, dix ans plus tard, c'est une bonne cinquantaine d'étudiants qui étaient inscrits à une quinzaine de cours. Si la session 2020 n'a pu être organisée pour les raisons que le monde connaît, la session 2021 a marqué la reprise des cours, avec le soutien indéfectible des Facultés FIAL et TECO, qui offrent les locaux, et la bienveillance du département GLOR (Langues et littératures anciennes), qui accorde un subside. Quant à l'aspect "recherche », Jean-Claude Haelewyck le concrétisa en 2012 par le lancement d'une publication électronique, le Bulletin de l'ABELAO ou $B A B E L A O^{2}$, qui fête donc cette année ses dix ans d'existence.

\footnotetext{
${ }^{1} \mathrm{http} / /$ www.abelao.eu.

${ }^{2}$ https://ojs.uclouvain.be/index.php/babelao/issue/archive.
} 
Après son accession à l'éméritat en 2017, le Professeur Haelewyck n'a cessé d'être sollicité pour assumer tel et tel cours non attribués, preuve que l'université peut difficilement se passer d'un enseignant compétent et polyvalent, qui prend un réel plaisir à donner cours et à s'investir pour ses étudiants.

\section{BIBLIOGRAPHIE THÉMATIQUE}

\section{A. Vieilles versions latines de la Bible}

\section{Esther}

1985 : J.-C. HAELEWYCK, « Le texte dit 'Lucianique' du livre d'Esther. Son étendue et sa cohérence », Le Muséon 98, p. 5-44.

1991 : J.-C. HAELEWYCK, «La version latine du livre d'Esther dans le Monacensis 6239. I. Texte », Revue Bénédictine 101, p. 7-27.

1993 : J.-C. HAELEWYCK, «La version latine du livre d'Esther dans le Monacensis 6239. II. Commentaire », Revue Bénédictine 103, p. 289-306.

1996 : J.-C. HAELEWYCK, «Les premières versions latines de la Bible », dans C.-B. AMPHOUX, J. MARGAIN (éd.), Les premières traditions de la Bible (Histoire du texte biblique. Studien zur Geschichte des biblischen Textes, 2), Lausanne, p. 121-136.

1999 : J.-C. HAELEWYCK, « La version latine du livre d'Esther dans la première Bible d'Alcalà. Avec un appendice sur les citations patristiques vieilles latines », dans J.-M. AUWERS, A. WÉNIN (éd.), Lectures et relectures de la Bible. Festschrift P.-M. Bogaert (Bibliotheca Ephemeridum Theologicarum Lovaniensium, 144), Louvain, p. 165-193.

1999 : J.-C. HAELEWYCK, « Le papyrus Oxyrhynque 4443 et la vetus latina du livre d'Esther », Revue Bénédictine 109, p. 267-271.

2003 : J.-C. HAELEWYCK, «L'anéantissement de l'autre. Réactions face à cet élément narratif dans la tradition grecque du livre d'Esther (textes o', $L$ et vetus latina) », dans J. RIAUD (éd.), Étrangers et exclus dans le monde biblique. Colloque International à l'Université Catholique de l'Ouest. Angers, les 21 et 22 février 2002 (coll. Théolarge, 3), Angers, p. 95-111.

2003 : J.-C. HAELEWYCK, Esther. Fasc. 1. Introduction (Vetus Latina. Die Reste der altlateinischen Bibel 7/3), Freiburg/Br., Herder, p. 1-80.

2004 : J.-C. HAELEWYCK, Esther. Fasc. 2. Introduction (suite et fin). Est A 1 - 2,7 (Vetus Latina. Die Reste der altlateinischen Bibel 7/3), Freiburg/Br., Herder, p. 81-160.

2006 : J.-C. HAELEWYCK, «The Relevance of the Old Latin Version for the Septuagint, with Special Emphasis on the Book of Esther », Journal of Theological Studies 57, p. 439- 473.

2006 : J.-C. HAELEWYCK, Esther. Fasc. 3. Est 2,7 - 4,7 (Vetus Latina. Die Reste der altlateinischen Bibel 7/3), Freiburg/Br., Herder, p. 161-240.

2008 : J.-C. HAELEWYCK, Esther. Fasc. 4. Est 4,8 - 6,2 (Vetus Latina. Die Reste der altlateinischen Bibel 7/3), Freiburg/Br., Herder, p. 241-320.

2008 : J.-C. HAELEWYCK, Esther. Fasc. 5. Est 6,3-F,11 (Vetus Latina. Die Reste der altlateinischen Bibel 7/3), Freiburg/Br., Herder, p. 321-440.

2017 : J.-C. HAELEWYCK, Esther Old Latin + Job Old Latin + Old Latin Additions to Esther, dans A. LANGE (éd.), The Textual History of the Bible, Leiden, p. 398-402, 860-864. 
2018 : J.-C. HAELEWYCK, « Nouveaux extraits d'Esther vieux latins (VL 155A) découverts dans la reliure du ms. Toulouse, Bibliothèque Municipale $158 »$, Revue Bénédictine 128, p. 77-83.

\section{Judith}

2019 : P. BogAert, J.-C. HAELEWyCK, Iudith. Fascicule 2. Iud 1,1-4,9 (Vetus Latina. Die Reste der altlateinischen Bibel 7/2), Freiburg/Br., Herder, p. 81-160.

2019 : P. BogAert, J.-C. HAELEWyCK, Iudith. Fascicule 3. Iud 4,9- 7,4 (Vetus Latina. Die Reste der altlateinischen Bibel 7/2), Freiburg/Br., Herder, p. 161-240.

2019 : P. Bogaert, J.-C. Haelewyck, Iudith. Fascicule 4. Iud 7,4 - 8,32 (Vetus Latina. Die Reste der altlateinischen Bibel 7/2), Freiburg/Br., Herder, p. 241-320.

2019 : P. Bogaert, J.-C. HAELEwyCK, Iudith. Fascicule 5. Iud 8,32 - 11,17 (Vetus Latina. Die Reste der altlateinischen Bibel 7/2), Freiburg/Br., Herder, p. 321-400.

2020 : P. Bogaert, J.-C. HaelewyCK, Iudith. Fascicule 6. Iud 11,17 - 14,19 (Vetus Latina. Die Reste der altlateinischen Bibel 7/2), Freiburg/Br., Herder, p. 401-480.

2020 : P. Bogaert, J.-C. HAelewyck, Iudith. Fascicule 7. Iud 14,19 - fin (Vetus Latina. Die Reste der altlateinischen Bibel 7/2), Freiburg/Br., Herder, p. 481-538.

\section{Isaïe}

1988 : J.-C. HAELEWYCK, «Le lemme vulgate du commentaire de Jérôme sur Isaïe », dans Y.-M. DuVAL (éd.), Jérôme entre l'Occident et l'Orient. XVIe centenaire du départ de saint Jérôme de Rome et de son installation à Bethléem. Actes du Colloque de Chantilly (Septembre 1986), Paris, p. 391-402.

1989 : J.-C. HAELEWYCK, «L'édition de la Vetus Latina d'Isaïe», dans J. VERMEYLEN (éd.), The Book of Isaiah. Le livre d'Isaïe. Les oracles et leurs relectures. Unité et complexité de l'ouvrage (Bibliotheca Ephemeridum Theologicarum Lovaniensium, 81), Louvain, p. 135-145.

1989 : J.-C. HAELEWYCK, « Le cantique 'De nocte'. Histoire du texte vieux latin d'Is. 26,9b- 20(21) », Revue Bénédictine 99, p. 7-34.

1989 : J.-C. HAELEWYCK, « Le cantique de la vigne. Histoire du texte vieux latin d'Isaïe 5,1- 7(9)», Ephemerides Theologicae Lovanienses 65, p. 257-279.

\section{Habacuc}

2021 : J.-C. HAELEWYCK, «Le texte vieux latin du cantique d'Habacuc 3,2-19 dans le Psautier de Vérone (VL 300) », Revue Bénédictine 131, p. 301-320.

— : J.-C. HAELEWYCK, « Le texte vieux latin du cantique d'Habacuc 3,2-19 dans les Libri canticorum annexés aux psautiers européens. Texte et commentaire », à paraître dans les Mélanges ChristianBernard Amphoux, Bruxelles, éditions Safran.

\section{Daniel}

2021 : J.-C. HAELEWYCK, Danihel. Fascicule 1 (Introduction, Dn 13,1-10) (Vetus Latina 14/1), Freiburg, p. 1-80.

2021 : J.-C. HaELEwYCK, Danihel. Fascicule 2 (Dn 13,11-64; 1,1 - 3,17) (Vetus Latina 14/1), Freiburg, p. 81-160.

2021 : J.-C. HAELEWYCK, Danihel. Fascicule 3 (Dn 3,18 - 4,24) (Vetus Latina 14/1), Freiburg, p. 161240. 
2021 : J.-C. HAELEWYCK, Danihel. Fascicule 4 (Dn 4,25 - 8,7) (Vetus Latina 14/1), Freiburg, p. 241320.

- : J.-C. HAELEWYCK, « Le cantique vieux latin d'Azarias (Dn 3,26-45) dans le manuscrit Verona, Biblioteca Capitolare IV fol. Édition et commentaire », à paraître dans Revue Biblique.

\section{6. Évangile de Marc}

1999 : J.-C. HAELEWYCK, « La version latine de Marc », Mélanges de Sciences religieuses 56, p. $27-$ 52.

2002 : C.-B. Amphoux, J.K. Elliott, J.-C. HAelewyck, « The Marc multilingue Project», Filologia Neotestamentaria 15, p. 3-17.

2003 : J.-C. HAELEWYCK, «La vetus latina de l'Évangile de Marc. Les rapports entre les témoins manuscrits et les citations patristiques », dans C.-B. AMPHOUX, J.K. ELLIOTT (éd.), The New Testament Text in Early Christianity. Proceedings of the Lille Colloquium, July 2000, Lausanne, Éditions du Zèbre, p. 151-193.

2005 : J.-C. HAELEWYCK, S. ARBACHE (éd.), Évangile de Marc. Les types de texte dans les langues anciennes, Lille (= Mélanges de Science Religieuse, 62).

2005 : J.-C. HAELEWYCK, «Marc 1,1-11. La tradition latine (vetus latina et vulgate) », Mélanges de Sciences religieuses, 62, p. 17-26.

2013 : J.-C. HAELEWYCK, « Un nouveau témoin vieux latin de Marc : le manuscrit Durham, Cathedral Library A. II. 10 + C. III. 03 + C. III. $20 »$, Revue Bénédictine 123, p. 5- 12.

2013 : J.-C. HAELEWYCK, Evangelium secundum Marcum. Fascicule 1. Introduction (Vetus Latina. Die Reste der altlateinischen Bibel 17), Freiburg/Br., Herder, p. 1-80.

2013 : J.-C. HAELEWYCK, Evangelium secundum Marcum. Fascicule 2. Introduction (fin) ; Mc 1,1-43 (Vetus Latina. Die Reste der altlateinischen Bibel 17), Freiburg/Br., Herder, p. 80-160.

2014 : J.-C. HAELEWYCK, Evangelium secundum Marcum. Fasicule 3. Mc 1,44 - 4,15 (Vetus Latina. Die Reste der altlateinischen Bibel 17), Freiburg/Br., Herder, p. 161-240.

2015 : J.-C. HAELEWYCK, Evangelium secundum Marcum. Fascicule 4. Mc 4,15 - 6,16 (Vetus Latina. Die Reste der altlateinischen Bibel 17), Freiburg/Br., Herder, p. 241-320.

2015 : J.-C. HAELEWYCK, Evangelium secundum Marcum. Fascicule 5. Mc 6,17 - 8,11 (Vetus Latina. Die Reste der altlateinischen Bibel 17), Freiburg/Br., Herder, 2015, p. 321-400.

2016 : J.-C. HAELEWYCK, Evangelium secundum Marcum. Fascicule 6. Mc 8,11 - 9,46 (Vetus Latina. Die Reste der altlateinischen Bibel 17), Freiburg/Br., Herder, p. 401-480.

2017 : J.-C. HAELEWYCK, Evangelium secundum Marcum. Fascicule 7. Mc 9,47 - 11,14 (Vetus Latina. Die Reste der altlateinischen Bibel 17), Freiburg/Br., Herder, p. 481-560.

2018: J.-C. HAELEWYCK, Evangelium secundum Marcum. Fascicule 8. Mc 11,14 - 13,5 (Vetus Latina. Die Reste der altlateinischen Bibel 17), Freiburg/Br., Herder, p. 561-640.

2018: J.-C. HAELEWYCK, Evangelium secundum Marcum. Fascicule 9. Mc 13,6 - 14,43 (Vetus Latina. Die Reste der altlateinischen Bibel 17), Freiburg/Br., Herder, p. 641-720.

2018 : J.-C. HAELEWYCK, Evangelium secundum Marcum. Fascicule 10. Mc 14,44 - fin (Vetus Latina. Die Reste der altlateinischen Bibel 17), Freiburg/Br., Herder, p. 721-842. 


\section{Actes des Apôtres}

1988 : J.-C. HAELEWYCK, «Le texte occidental des Actes des Apôtres. À propos de la reconstitution de M.-E. Boismard et A. Lamouille », Revue Théologique de Louvain 19, p 343-353.

1993 : J.-C. HAELEWyCK, P.-A. Deproost, « Le texte biblique des Actes et l'authenticité des sommaires en prose dans l'Historia apostolica d'Arator», dans R. GRYSON (éd.), Philologia sacra. Biblische und patristischen Studien für Hermann J. Frede und Walter Thiele zu ihrem siebzigsten Geburtstag, 2 Bände (Aus der Geschichte der lateinischen Bibel, 24/1. 2), Freiburg/Br., Herder, p. 583-604.

\section{B. Traditions davidiques}

1994 : J.-C. HAELEWYCK, « Le meurtre d'Asaël, une péripétie de la bataille de Gabaon (II Sam 2, $12-$ 32) ? », Zeitschrift für die alttestamentliche Wissenschaft 106, p. 27-39.

1995 : J.-C. HAELEWYCK, « David a-t-il régné du vivant de Saül ? Étude littéraire et historique de II Sm 2, 1-11», Revue Théologique de Louvain 26, p. 165-184.

1995 : J.-C. HAELEWYCK, «La mort d'Abner : 2 Sam 3, 1-39. Étude littéraire et historique », Revue Biblique 102, p. 161-192.

1997 : J.-C. HAELEWYCK, «L'assassinat d'Ishbaal (2 Sm IV 1-12)», Vetus Testamentum 47, p. 145153.

\section{Apocryphes de l'Ancien Testament}

1998 : J.-C. HAELEWYCK, Clavis Apocryphorum Veteris Testamenti (Corpus Christianorum), Turnhout, Brepols, $18+243$ p.

2000 : A.-M. DENIS (avec le concours de J.-C. HAELEWYCK), Introduction à la littérature religieuse judéo-hellénistique. Pseudépigraphes de l'Ancien Testament, 2 vol., Turnhout, Brepols, 1420 p.

2002 : J.-C. HAELEWYCK, « La reine de Saba et les apocryphes salomoniens (Testament de Salomon et Questions de la reine de Saba) », Graphè 11, p. 83-99.

2011 : J.-C. HAELEWYCK, «Le nombre des Rois Mages. Les hésitations de la tradition syriaque », dans J.-M. VERCRUYSSE, Les (Rois) Mages, (coll. Graphè, 20), Arras, p. 25- 37.

2013 : J.-C. HAELEWYCK, « Narratio Zosimi de Vita Beatorum (CAVT 166). Une relecture du mythe de l'Île des Bienheureux », dans C. CANNUYER (dir.), L'île, regards orientaux. Varia orientalia, biblica et antiqua Hans Hauben in honorem (Acta Orientalia Belgica, XXVI), Lille, p. 135-147.

2014 : J.-C. HAELEWYCK, «Historia Zosimi de Vita Beatorum Rechabitarum. Édition de la version syriaque brève », Le Muséon 127, p. 95-147.

2015 : J.-C. HAELEWYCK, «La version syriaque longue de l'Historia Zosimi de Vita Beatorum Rechabitarum. Édition et traduction », Le Muséon 128, p. 295-379.

2016 : J.-C. Haelewyck, V. Somers, E. VAn Elverdinghe, « Diverse Perspective on the Manuscript Tradition of the Story of Zosimus », Oriens Christianus 99, p. 1-44.

2016 : J.-C. HAELEWYCK (dir.), Histoire de Zosime sur la Vie des Bienheureux Réchabites. Les versions orientales et leurs manuscrits, (Corpus Scriptorum Christianorum Orientalium, 664. Subsidia, 135), Louvain, Peeters, $180 \mathrm{p}$.

2017 : J.-C. HAELEWYCK, «Histoire de Zosime sur la vie des Bienheureux Réchabites. Les trois recensions syriaques. Édition de la recension résumée », Parole de l'Orient 43, p. 175-194. 


\section{Grégoire de Nazianze}

2001 : J.-C. HAelewyCK, Sancti Gregorii Nazianzeni Opera. Versio syriaca I. Oratio 40 (Corpus Christianorum. Corpus Nazianzenum, 14), Turnhout, Brepols, $40+221$ p.

2007 : J.-C. HAELEWYCK, Sancti Gregorii Nazianzeni Opera. Versio syriaca IV. Orationes 28, 29, 30 et 31 (Corpus Christianorum. Corpus Nazianzenum, 23), Turnhout, Brepols, $14+407 \mathrm{p}$.

2011 : J.-C. HAELEWYCK, Sancti Gregorii Nazianzeni Opera. Versio syriaca V. Orationes 1, 2, 3 (Corpus Christianorum. Corpus Nazianzenum, 25), Turnhout, Brepols, $16+217 \mathrm{p}$.

2016 : J.-C. HAELEWYCK, « Le Discours 40, 1-2 de Grégoire de Nazianze dans les trois versions syriaques. D'une traduction libre à une traduction miroir », dans V. SOMERS, P. YANNOPOULOS (éd.), Philokappadox. In Memoriam Justin Mossay (Orientalia Lovaniensia Analecta, 251), p. 183-195.

2017 : J.-C. HAELEWYCK, « Les versions syriaques des Discours de Grégoire de Nazianze : un processus continu de révision », BABELAO 6, p. 75-124.

2018 : B. KindT, J.-C. HAElEwYCK, A. SChMidT, N. AtAS, « La concordance bilingue grecquesyriaque des Discours de Grégoire de Nazianze », BABELAO 7, p. 51-80.

\section{E. Langues sémitiques}

2004 : J. WeInGReEN, Hébreu biblique. Méthode élémentaire, $2^{\mathrm{e}}$ édition revue et corrigée par J.-C. HAELEWYCK, Paris, Beauchesne, $307 \mathrm{p}$.

2006 : J.-C. HAELEWYCK, Grammaire comparée des langues sémitiques. Éléments de phonétique, de morphologie et de syntaxe (Langues et cultures anciennes, 7), Bruxelles, Safran, $191 \mathrm{p}$.

2008 : J.-C. HAELEWYCK, «L'inscription phénicienne du sarcophage d'Ahiram. Un essai de vocalisation », Res Antiquae 5, p. 439-450.

2011 : J.-C. HAELEWYCK, « L'inscription phénicienne de Tabnit (KAI 13). Essai de vocalisation », Res Antiquae 8, p. 1-12.

2012 : J.-C. HAELEWYCK, «The Phoenician Inscription of Eshmunazar. An Attempt at Vocalization », BABELAO 1, p. 77-98.

2015 : J.-C. HAELEWYCK, Grammaire comparée des langues sémitiques. Éléments de phonétique, de morphologie et de syntaxe. Deuxième édition entièrement revue (Langues et cultures anciennes, 24), Bruxelles, Safran, 199 p.

\section{F. Critique textuelle du Nouveau Testament}

2013 : J.-C. HAELEWYCK, « The Healing of a Leper (Mark 1,40-45). A Textual Commentary », Ephemerides Theologicae Lovanienses 89, p. 15-36.

2014 : J.-C. HAELEWYCK, « Les versions anciennes », dans C.-B. AMPHOUX (éd.), Manuel de critique textuelle du Nouveau Testament, 1. Introduction générale (Langues et cultures anciennes, 22), Bruxelles, Safran, p. 75-144.

2016 : J.-C. HAELEWYCK, «'Un enseignement nouveau donné d'autorité'. Remarques de critique textuelle sur l'épisode de la guérison du démoniaque en Mc 1,23-27 », dans Th.-J. BAUER (éd.), Traditio et Translatio. Studien zur lateinischen Bibel zu Ehren von Roger Gryson, (Vetus Latina. Die Reste der altlateinischen Bibel nach Petrus Sabatier neu gesammelt, herausgegeben von der Erzabtei Beuron. Aus der Geschichte der lateinischen Bibel 40), Freiburg im Breisgau, p. 99-115.

2017 : J.-C. HAELEWYCK (éd.), Le Nouveau Testament syriaque (Études syriaques, 14), Paris, Geuthner, XII + 342 p. 
2017 : J.-C. HAELEWYCK, « Les Vieilles versions syriaques des Évangiles », dans J.-C. HAELEWYCK (éd.), Le Nouveau Testament syriaque (Études syriaques, 14), Paris, Geuthner, p. 67-113.

2019 : J.-C. HAELEWYCK, «The Old Syriac Versions of the Gospels. A Status Quaestionis (From 1842 to the Present Day)», BABELAO 8, p. 141-179.

2019 : J.-C. HAELEWYCK, « “Sub Abiathar principe sacerdotum”. Réflexion sur la méthode en critique textuelle à partir de l'épisode des épis arrachés en Mc 2,23-28 », Revue Biblique, 126, p. 401-414.

2022 : J.-C. HAELEWYCK, «L'institution des Douze en Mc 3,13-16a. Un cas paradigmatique en critique textuelle du Nouveau Testament grec », dans C. CANNUYER (éd.), Orients pluriels. Liber amicorum Samir Arbache (Acta Orientalia Belgica, XXXV), Bruxelles, Lille, p. 41-53.

\section{G. Divers}

1985 : J.-C. HAELEWYCK, «Introduction à l'Ancien Testament. À propos de quelques publications récentes », Revue Théologique de Louvain 16, p. 455-463.

1995 : G. GanglOFF, J.-C. HaElewYCK, «Osée 4, 17-19. Un marzeah en l'honneur de la déesse Anat? », Ephemerides Theologicae Lovanienses 71, p. 370-382.

2008 : J.-C. HAELEWYCK, «Le canon de l'Ancien Testament dans la tradition syriaque (manuscrits bibliques, listes canoniques, auteurs)», dans F. BriQuel Chatonnet, Ph. Le Moigne (éd.), L'ancien Testament en syriaque (Études syriaques, 5), Paris, p. 141-171.

2010 : J.-C. HAELEWYCK, «L'apport des Instituta de Junillus Africanus à la question du canon scripturaire de la tradition syriaque (Ancien Testament) », dans F. BRIQUEL CHATONNET, M. DEBIÉ (éd.), Sur les pas des Araméens chrétiens. Mélanges offerts à Alain Desreumaux (Cahiers d'Études Syriaques, 1), Paris, p. 25-34.

2016 : J.-C. HAELEWYCK, " Megiddo dans la Bible : mille ans d'histoire, de Josué à l'Apocalypse » dans C. Karlshausen, Cl OBSOMER (éd.), De la Nubie à Qadech. La guerre dans l'Égypte ancienne / From Nubia to Kadesh: War in Ancient Egypt (Connaissance de l'Égypte ancienne, 17), Bruxelles, p. 43-61.

2020 : J.-C. HAELEWYCK, B. KINDT (éd.), Le livre de Jérémie en perspective. Les deux rédactions conservées et l'addition du supplément sous le nom de Baruch. Recueil des travaux de PierreMaurice Bogaert (Bibliotheca Ephemeridum Theologicarum Lovaniensium, 308), Leuven, Paris, Bristol, Peeters, 535 p. 